\title{
Recovery of function after brain damage: On withdrawals from the memory bank
}

\author{
T. E. LeVERE, N. D. LeVERE, E. T. CHAPPELL, and P. HANKEY \\ North Carolina State University, Raleigh, North Carolina
}

\begin{abstract}
The present research addressed the question of whether any change between the conditions of original preoperative learning and postoperative recovery would interfere with the availability and retrieval of the spared memories. The procedure was to train rats initially on a brightness discrimination for appetitive rewards and then postoperatively reinstate the appetitive motivation but require that the animals recover the brightness habit, or its reversal, to escape/avoid footshock. The postoperative availability of the spared memories of the preoperative learning was quantified by the occurrence of impaired postoperative reversal training. The results indicated that simply reinstating the preoperative motivation state is insufficient to allow the animal access to the preoperative memories. However, if the preoperative mótivation state is reinstated and the animal is also provided rewards appropriate to that motiyation, then the addition of another motivation and rewards will not prevent the retrieval of spared preoperative memories.
\end{abstract}

The fact that visual decorticate rats are severely impaired when trained on the reversal of a preoperatively learned brightness discrimination demonstrates that this neocortical injury does not destroy the memories of the preoperatively learned behavior even though the lesioned animal is initially unable to perform the task (T. E. LeVere \& Morlock, 1973, 1974). It would appear that lesions to other neocortical areas also do not destroy the memories of preoperatively learned behaviors. For example, somatosensory lesions can disrupt the performance of a learned tactual discrimination even though reversal training will demonstrate that the memory of the behavior is spared (Weese, Neimand, \& Finger, 1973). Thus it would seem that an important question, if not the important question, for recovery of function theory is why the lesioned animal does not avail itself of memories that are spared. The present research was concerned with this question and what determines the postoperative availability of the spared memories of preoperatively learned behaviors.

We believe that part of the answer to this question rests with the animal's attempt to compensate for localized neural injury by utilizing noninjured neural systems (see T. E. LeVere, 1980). If, for example, the visual decorticate rat is trained with visual/nonvisual compound cues, and thus provided the opportunity to compensate for its brain injury by using nonvisual cues, then it will perform significantly better than even normal rats but not recover anything of a preoperatively learned brightness habit (T. E. LeVere \& N. D. Levere, 1982). On the other hand, if

This research was supported by Research Grant NS-12459 from the National Institute of Neurological and Communicative Disorders and Stroke, HHS to T. E. LeVere. The authors' mailing address is: Neuropsychology Laboratory, Department of Psychology, North Carolina State University, Raleigh, NC 27650. postoperative learning, that is, attempted compensation, is prevented by drugs, then the postoperative recovery of a preoperative brightness habit will be facilitated (Davis \& T. E. LeVere, 1979).

It would seem, on the basis of these data, that the availability of spared preoperative memories simply depends upon whether the lesioned animal utilizes the neural system with which the memories are associated. Unfortunately, this is not quite so. For example, the availability of spared memories can be demonstrated by the fact that these memories interfere with postoperative reversal training (T. E. LeVere \& Davis, 1977; T. E. LeVere \& Morlock, 1973, 1974). However, there is no such interference during postoperative reversal training if the conditions of recovery training are different from the conditions of original learning (T. E. LeVere \& Davis, 1977). Apparently, simply utilizing what remains of an injured neural system is not always sufficient to enable the lesioned animal to retrieve the memories associated with that system. It would seem that there also must be a high degree of similarity between the conditions of preoperative learning and postoperative recovery. Our question in the present investigations was whether this similarity need be absolute.

\section{EXPERIMENT 1}

We first addressed this question by using a variation of the T. E. LeVere and Davis (1977) experiment, which demonstrated that changing motivation between preoperative learning and postoperative recovery prevents the retrieval of spared memories (T. E. LeVere \& Davis, 1977). Initially we trained rats on a brightness discrimination for food reward. During postoperative recovery, the animals were again food deprived but now rewarded for correct responses by the termination of mild footshock. 
Thus, the present experiment was identical to the earlier research (T. E. LeVere \& Davis, 1977), except that we reinstated the motivational state of the preoperative learning during postoperative recovery training.

Our predictions with this paradigm were quite simple. If the similarity between original preoperative learning and postoperative recovery needs to be absolute for the postoperative retrieval of spared preoperative memories, then the change to postoperative shock reward should prevent their retrieval. In this case, we would replicate our earlier findings (T. E. LeVere \& Davis, 1977), and the lesioned animals should recover the original discrimination and the reversal of the original discrimination with equal efficiency. On the other hand, if the similarity does not need to be absolute, then reinstating the preoperative motivational state during postoperative recovery training might be expected to facilitate the retrieval of the spared preoperative memories. In this case, we would not replicate our earlier findings, and there should be a significant impairment associated with postoperative reversal training.

\section{Method}

Subjects. The subjects were 57 male hooded rats between the ages of 90 and 120 days. The animals were raised in our laboratory colony and maintained in group cages on a reversed 10:14-h day:night schedule. The animals were transferred to individual cages after being randomly selected for participation in the experiment. During training, all animals were $23 \mathrm{~h}$ food-deprived.

Apparatus. The apparatus was our Rat Automated Training System (RATS), previously described in detail by T. E. LeVere and Fontaine (1978). Basically, RATS is an equilateral Y-maze with walls and ceiling constructed of plastic and a floor made of brass rods. The rear wall of each arm of the maze is translucent plastic and can be back-lighted by a dc light bulb. The brightness cues are produced by powering these bulbs with different voltages to produce $0.5 \mathrm{fL}$ for the dim cue and $30.0 \mathrm{fL}$ for the bright cue, as measured from the entrance to the arm. The rat responds in this apparatus by running into the alley with the correct brightness and interrupting a photoelectric beam across the alley some 2 in. from the rear wall.

For food-rewarded training, RATS was fitted with commercially available pellet dispensers at the end of each alley. If the rat's response was correct, then three Noyes food pellets were dropped into a cup attached to the translucent rear panel. Responses to the incorrect alley were not rewarded. For postoperative shockmotivated training, the animal was required to avoid/escape pulsating footshock ( $0.5 \mathrm{sec}$ on $/ 0.5 \mathrm{sec}$ off) by running to the correct alley. If the responses were incorrect, the pulsating shock became steady until the error was corrected.

Procedure. All rats were preoperatively trained on the brightness discrimination for food reward. Half of the animals $(n=29)$ were trained with the brighter cue's indicating the correct alley, and half were trained with the dimmer cue's indicating the correct alley $(\mathrm{n}=28)$. All animals were trained for 10 trials each day, 7 days per week, and, within $48 \mathrm{~h}$ of reaching criterion ( 9 firstchoice correct responses within a day's training), subjected to visual decortication. Following visual decortication, each animal was allowed a 2-week rest with free access to food and water during the first 11 days of this period.

Visual decortication was accomplished by aspiration under sodium pentobarbital anesthesia. The lesions were intended to include all neocortex receiving thalamic projections (Hughes, 1977), and thus all neocortex posterior to a line beginning at bregma and be- coming tangential to the rhinal sulcus was removed. Following surgery, all animals received an intramuscular prophylactic injection of a broadband antibiotic.

The animals were assigned to one of four postoperative training conditions on the basis of preoperative errors to criterion to control for the effects of original acquisition. Fifteen of the animals of the preoperatively bright-correct group were postoperatively retrained on the original brightness discrimination; the remaining 14 animals were retrained on the brightness discrimination but with the correct cue being the opposite of what had been preoperatively learned. Similarly, 14 of the originally dim-correct group were retrained on the originally learned preoperative brightness discrimination; the remaining 14 animals were retrained on the reversal of the original problem.

Three days prior to postoperative training, each animal was again put on the 23-h food-deprivation regimen used during preoperative training. On the 15th postoperative day, each animal began recovery training to avoid/escape footshock. However, even though the animals were food-deprived, no food reinforcement was given for correct choices. Our interest here, of course, was whether reinstating the animal's preoperative motivational state would be sufficient to enable it to access the spared memories of the preoperatively learned behavior. If it was, then the animals trained on the reversal problem should show a significant impairment relative to the animals required to recover the originally learned brightness discrimination.

As each animal completed postoperative training, it was again anesthetized with sodium pentobarbital and perfused with saline followed by buffered Formalin. After the brain was removed, the extent of the lesion was drawn on surface diagrams with the aid of a dissecting microscope. Following this, the brain was sunk in a sucrose solution and cut into 50-micron frontal sections with a freezing microtome. Every 5th section through the neocortical lesion and the dorsal lateral geniculate nucleus was saved and stained with cresyl violet.

\section{Results}

Histology. Surface diagrams of the rats sustaining the smallest and largest visual lesions are shown in Figure 1. An ANOVA failed to suggest that lesion sizes make any difference between the animals postoperatively retrained with the bright or dim cue correct or between those retrained on the nonreversal or the reversal problem $(\mathrm{F}$ $<1$ in all cases).

Microscopic analysis of cresyl-violet-stained frozen sections indicated that all six neocortical layers were destroyed. In no instance was there bilateral invasion of any subcortical structures. The dorsal lateral geniculate

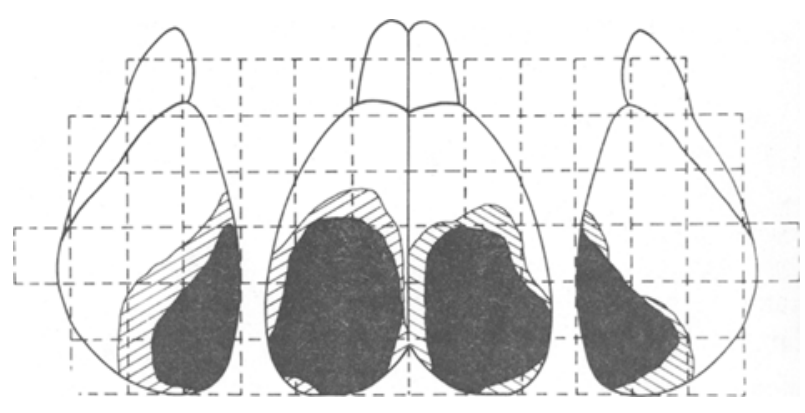

Figure 1. Surface diagrams indicating the smallest (black) and largest (cross-hatched) lesions sustained by the animals of this experiment. 


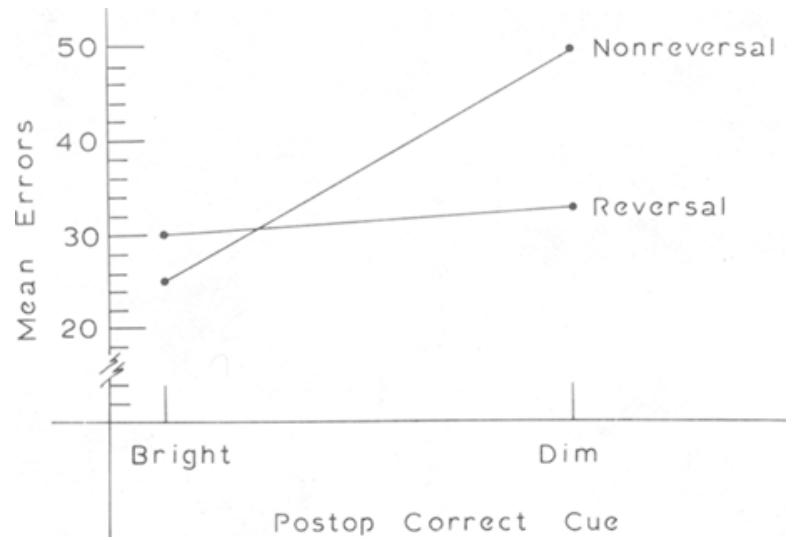

Figure 2. Mean errors to criterion during postoperative recovery for the nonreversal- and reversal-trained animals when the bright cue was correct and when the dim cue was correct.

nucleus showed complete retrograde degeneration, and some degeneration was evident in the posterior lateral nucleus, although this was not extensive. The ventral lateral geniculate nucleus appeared normal.

Behavior. Since the animals were intentionally matched, the mean preoperative error scores of 21.5 and 22.3 for the animals assigned to the nonreversal and reversal postoperative groups were not significantly different.

The mean error scores during postoperative nonreversal and reversal training are presented in Figure 2. As is apparent from the figure, requiring some of the animals to acquire the reversal of the preoperative problem did not differentially affect their performance, which means that the memories of what was learned preoperatively did not influence postoperative behavior. Or, put otherwise, postoperatively reinstating the animal's preoperative motivational state apparently is not sufficient to allow the lesioned rat to retrieve the memories associated with this motivational state.

A two-way ANOVA with main factors of correct cue (bright vs. dim) and postoperative discrimination problem (nonreversal vs. reversal) indicated a significant interaction between the main effects $[F(1,53)=7.28]$, whereas only the correct cue main effect reached acceptable levels of confidence $[F(1,53)=10.54$ and $F(1,53)=2.06$, respectively, for correct cue and discrimination problem].

\section{EXPERIMENT 2}

The results of Experiment 1 suggest that simply reestablishing the motivational state of a preoperative behavior is not sufficient to make the memory of this behavior available during postoperative recovery training. Accordingly, in a second experiment we tried another strategy. The procedure was a replication of Experiment 1, in which the animals were preoperatively trained for food reward and postoperatively food-deprived and shock-motivated. However, in the present experiment, in addition to avoiding/escaping footshock, the animals were provided food rewards for correct responses. Our purpose here was to determine the degree to which the postoperative situation would have to duplicate the original preoperative learning conditions in order for these memories to be available during recovery training. If all that was necessary was to reinstate the conditions of preoperative learning situation per se, then the appetitive motivation and food reward should allow for retrieval of preoperative memories and there should be a significant postoperative reversal impairment. On the other hand, if it was necessary to faithfully duplicate the conditions of the preoperative learning situation, then the addition of shock escape motivation and reward might be expected to lessen the availability of spared preoperative memories and there should be no postoperative reversal impairment.

\section{Method}

Subjects. Forty male hooded rats between the ages of 90 and 120 days served as subjects. The animals were treated as described for Experiment 1.

Apparatus. We again used the RATS apparatus.

Procedure. The procedure was an exact replication of Experiment 1 with the sole exception that during postoperative training the animals were given three food pellets for each correct choice in addition to the reward of escaping footshock. Preoperatively, 20 rats were trained with the bright cue correct and 20 were trained with the dim cue correct. After surgery and a 2-week rest, 10 of the bright-cue-correct group were retrained on the original problem and the remaining 10 were trained on the reversal of the original problem. The dim-cue-correct group was similarly divided. As in Experiment 1, the animals were assigned to postoperative nonreversal and reversal groups on the basis of their preoperative learning scores (errors to criterion), so that the postoperative groups would be matched. Also, like Experiment 1, our primary interest was the postoperative availability of the memories of the preoperatively learned behavior, so our major consideration was the performance of postoperative nonreversal and reversal groups in terms of errors required to regain $9 / 10$ criterion performance.

\section{Results}

Histology. Histological examination revealed essentially the same results as those reported for Experiment 1. Surface diagrams of the smallest and largest visual lesions are presented in Figure 3.

Behavior. We were again successful in matching the postoperative groups and found no significant differences between the preoperative performances of the animals as-

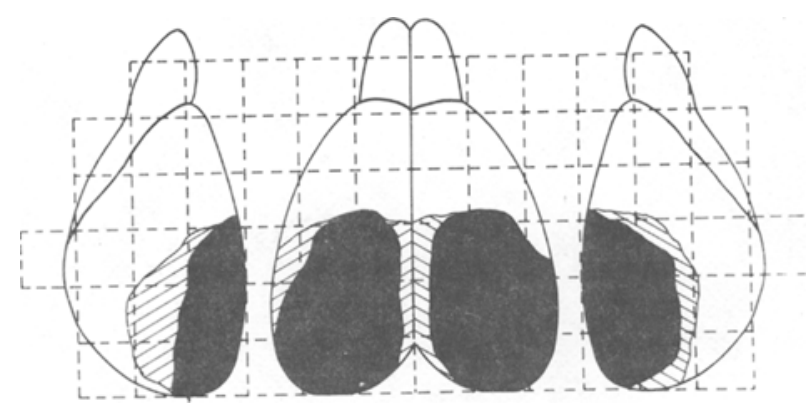

Figure 3. Surface diagrams of the smallest (black) and largest (cross-hatched) lesions for the animals of Experiment 2. 
signed to the nonreversal groups (mean errors to criterion $=22.1$ ) and the reversal groups (mean errors to criterion $=26.0$ ).

Figure 4 presents the postoperative mean error scores. Again, as in Experiment 1, there was no consistent effect of reversal training, indicating that providing rewards appropriate to the reinstated preoperative motivational state was not sufficient to make the preoperative memories available when the animals were also responding to avoid shock. Statistically, a two-way ANOVA using main factors of brightness of correct cue and postoperative discriminative problem indicated no significant main effects or interaction $[\mathrm{F}(1,36)=2.23, \mathrm{~F}(1,36)=0.45$, and $\mathrm{F}(1,36)=2.35$, respectively].

However, before accepting this result, it is important to remember that simply providing food pellets does not assure that the rats will respond to this reward and eat the pellets; and some did not. Specifically, 6 of the 20 nonreversal trained rats and 8 of the 20 reversal trained rats did not eat the food pellets on at least $90 \%$ of their postoperative training days. Put another way, slightly over one-third of the rats did not conform to the design of the experiment, which, of course, severely compromised the hypothesis under test.

Because of this, we analyzed the data excluding the rats that did not eat the food pellets. These data are presented in Figure 5, which shows a strong and consistent impairment associated with postoperative reversal training. In fact, the statistics are somewhat impressive considering that we were able to use only two-thirds of the original subject population. That is, a two-way ANOVA revealed that the main effect of postoperative problem type, that is, nonreversal versus reversal, was now significant $[F(1,22)=6.15]$, whereas the brightness main effect and the interaction did not reach significance $[F(1,22)=1.06$ and $F(1,22)=0.48$, respectively]. We also performed an ANOVA using the subjects that did not eat the food rewards, and found no significant main effects or interaction ( $\mathrm{F}<1$ in all cases). Although this null result sup-

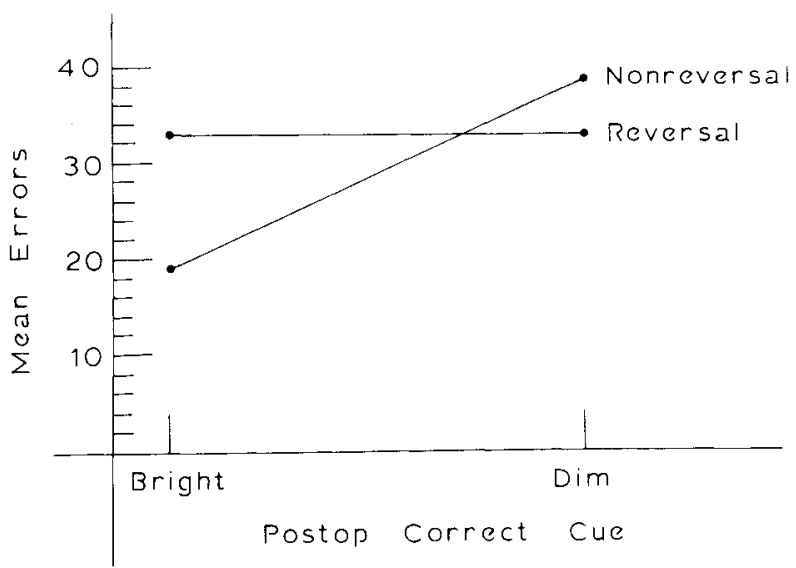

Figure 4. Mean errors to criterion for all animals during postoperative recovery for nonreversal- and reversal-trained animals when the bright cue was correct and when the dim cue was correct.

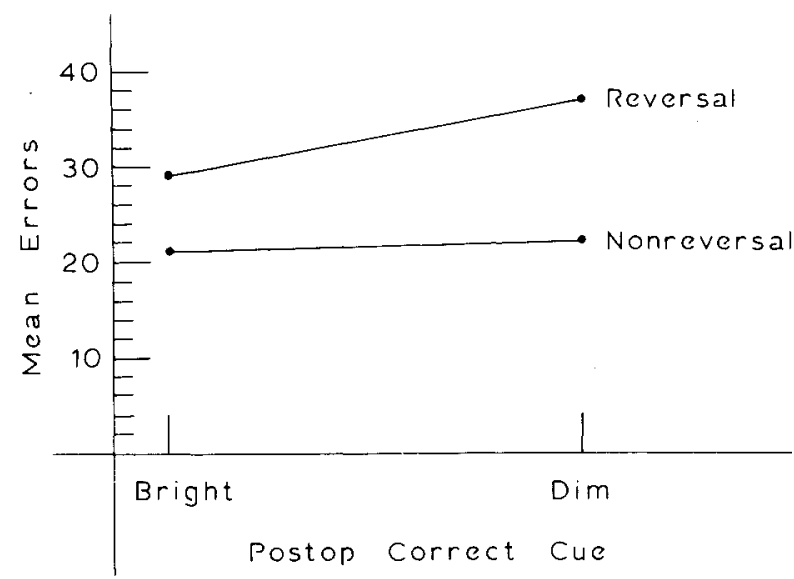

Figure 5. Same as Figure 4 but including only those animals that ate the available food rewards.

ports our finding with the animals that did eat, it must be viewed with some caution because of the small number of subjects. Nevertheless, the fact still remains that if the conditions of preoperative original learning are met during postoperative recovery training, then the preoperatively established memories are available to the visual decorticate rat and will influence the progress of recovery of function. And they will do so even when there is not complete parity between the preoperative and postoperative training situations.

One final consideration is whether the animals that ate the food reward also received the shock motivation. This is an issue, because food-reward training is subject-paced in RATS and there is no imposed intertrial interval. However, during shock-motivated training, there is a 15$\mathrm{sec}$ intertrial interval and the animal is allowed a $10-\mathrm{sec}$ shock-free period after the onset of the discriminative cues at the beginning of each trial. Thus, if an animal was responding quickly to obtain food reward, it would be possible for it to avoid footshock completely, and this would invalidate our result just as much as the rats' not eating the provided food rewards. This was not the case, however, since the nonreversal group averaged only $19.3 \%$ avoidances and the reversal group averaged only $16.7 \%$ avoidances. Clearly, whatever the effects of the shock motivation were, the animals were not ignorant of its presence.

\section{DISCUSSION}

The present research was concerned with the determinants of the availability of the memories of preoperatively learned behaviors following brain injury. On the basis of our own research and the extensive summary of Meyer and Meyer (1982), we were persuaded that the issue reduced to a simple question of parity between the conditions of preoperative learning and postoperative recovery. Recent evidence, however, suggests that this simple proposition is in need of some qualification, if not revision. 
For example, T. E. LeVere, Chappell, and N. D. LeVere (1984) have suggested that all memories are not the same and that the memory itself can determine whether the lesioned animal will retrieve the spared preoperative memory. It would appear that more general memories, for example, memories established to multiple motivational states, are more likely to be available during postoperative recovery than are the memories of similar behaviors learned to satisfy a single motivational state. Thus, the conditions under which a memory is stored can be an important determinant of the availability of this memory during postoperative recovery. Our question in the present research was whether the conditions under which a memory was retrieved were similarly important.

On one level, the answer to this is already available. T. E. LeVere and Davis (1977) have demonstrated that changing the motivational state between preoperative learning and postoperative recovery can interfere with the retrieval of certain spared preoperative memories. Morcover, on the basis of classical transfer-of-training experiments (Osgood, 1949), it would seem to be a trivial exercise to change sufficiently the conditions between learning and remembering so that the latter was not likely to occur. Perhaps so, but this then begs the question, particularly with regard to understanding the consequences of brain injury. The issue, in this case, is not whether a sufficient change can make a preoperatively established memory unavailable following brain injury, but, rather, whether any change will do so. As noted above, we were previously of the mind that any change between preoperative learning and postoperative recovery training would disallow access to these memories. However, the data now indicate that this is not quite so for more generalized memories, and the present results suggest that the position is not as invariant as we had originally thought, even for more limited memories.

Specifically, the present research shows that it is possible to change the conditions of postoperative recovery and still retrieve spared memories provided one condition is met. This condition is that the change must be additive in the sense that it does not disrupt or replace the conditions of original learning. In other words, we stand by our earlier assertion that the postoperative availability of singly motivated behaviors critically depends on reestablishing the conditions under which the original learning occurred. But it would seem that, once this is accomplished, the brain-injured rat is tolerant of other recovery conditions.

It must be emphasized, however, that the original learning conditions must be faithfully reinstated during the postoperative training procedure. Simply reinstating some subset of the preoperative conditions, for example only the animal's preoperative motivational state (Experiment 1), is insufficient, and the memories of the preoperative behavior will not be available to the animal when it is rewarded for some additional motivation. In fact, even providing reinforcement appropriate to the reinstated preoperative motivational state, that is, replicating all of the preoperative learning conditions, may not be sufficient if the animal does not respond to these conditions (Experiment 2). The principle would seem to be that if the original learning situation is recreated during postoperative recovery, and if the brain-injured animal responds to this situation, then the addition of other postoperative conditions may be relatively unimportant to the retrieval of spared preoperative memories.

We suggest that this latter point has important pragmatic considerations for postoperative therapy. In this regard, the most important factor in the postoperative availability of what is spared is the parity between the intentions of the therapist and the actions of the brain-injured subject, since, in the final analysis, it is how the subject responds, not what the therapist wishes, which determines the conditions under which recovery of function is attempted. In other words, from the point of view of recovery of function, the conditions of the withdrawal determine what may be obtained from the memory bank following brain injury.

\section{REFERENCES}

DAVIS, N., \& LEVERE, T. E. (1979). Recovery of function after brain damage: Different processes and the facilitation of one. Physiological Psychology, 7, 233-240.

HugHes, H. G. (1977). Anatomical and neurobehavioral investigations concerning the thalamo-cortical organization of the rat's visual system. Journal of Comparative Neurology, 175, 311-335.

LEVERE, T. E. (1980). Recovery of function after brain damage: A theory of the behavioral deficit. Physiological Psychology, 8, 279-308.

LeVere, T. E., Chappeld, E. T., \& LeVere, N. D. (1984). Recovery of function after brain damage: On deposits to the memory bank. Physiological Psychology, 12, 209-212.

LEVERE, T. E., \& DAVIS, N. (1977). Recovery of function after brain damage: The motivational specificity of spared neural traces. $E x$ perimental Neurology, 57, 883-889.

LEVERE, T. E., \& FontAine, C. W. (1978). A demonstration of the importance of RNA metabolism for the acquisition but not performance of learned behaviors. Experimental Neurology, 59, 444-449.

LEVERE, T. E, \& LEVERE, N. D. (1982). Recovery of function after brain damage: Support for the compensation theory of the behavioral deficit. Physiological Psychology, 10, 165-174.

LeVere, T. E., \& Morlock, G. W. (1973). The nature of visual recovery following posterior neodecortication in the hooded rat. Joumal of Comparative and Physiological Psychology, 83, 62-67.

LEVERE, T. E., \& MORLOCK, G. W. (1974). The influence of preoperative learning on the recovery of a successive brightness discrimination. Bulletin of the Psychonomic Society, 4, 507-509.

MeYer, P. M., \& MEYER, D. R. (1982). Memory, remembering, and amnesia. In R. L. Isaacson \& N. E. Spear (Eds.), The expression of knowledge. New York: Plenum.

OsGOOD, C. E. (1949). The similarity paradox in human learning: A resolution. Psychological Review, 56, 132-143.

Weese, G. D., Neimand, D., \& Finger, S. (1973). Cortical lesions and somesthesis in rats: Effects of training and overtraining prior to surgery. Experimental Brain Research, 16, 542-550.

(Manuscript received January 28, 1984; revision accepted for publication September 8,1984 .) 